\title{
Accelerated 3D self-gated cardiac cine imaging at 3T using a tiny golden angle and compressed sensing
}

\author{
Xiaoyong Zhang ${ }^{1,2^{*}}$, Guoxi Xie ${ }^{2}$, Hanwei Chen ${ }^{3}$, Yanchun Zhu², Zijun Wei ${ }^{2}$, Shi Su², Fei Yan², Bensheng Qiu', \\ Xin Liu'2, Zhaoyang Fan ${ }^{4}$ \\ From 19th Annual SCMR Scientific Sessions \\ Los Angeles, CA, USA. 27-30 January 2016
}

\section{Background}

3D self-gated (SG) cine imaging with TrueFISP not only provides excellent contrast between myocardium and blood, but also eliminates the need for ECG set up and permits free-breathing acquisitions [1]. However, such Cartesian sampling-based techniques are commonly used at $1.5 \mathrm{~T}$ due to the eddy current and SAR problems as well as time-consuming on data acquisition under the Nyquist sampling criteria. To achieve time-efficient $3 \mathrm{~T}$ cine imaging, a novel accelerated SG method, named SparseSG, was proposed using a tiny golden angle and compressed sensing [2].

\section{Methods: Sequence}

A 3D hybrid radial sampling pattern was adopted for the SparseSG [1]. In order to reduce the eddy current effect, a tiny golden angle of $32.039^{\circ}$, instead of $111.246^{\circ}$, was used for data acquisition (Figure 1). After the respiratory and cardiac motions were determined by processing the SG data as [1], the acquired data was retrospectively sorted into different respiratory and cardiac phases. A compressed sensing method exploiting the image sparsity in k-t space was used for image reconstruction, thus effectively shortening the scan time and reducing SAR.

\section{Experiment}

IRB-approved cardiac imaging was performed on 5 healthy subjects (2M, 3F, age 20 26) at $3 \mathrm{~T}$ (Siemens Tim Trio, Germany) with a standard 6-channel body coil and a spine coil. Scan parameters included: 3D imaging with standard short-axis, TR $=3.8 \mathrm{~ms}, \mathrm{TE}=1.9 \mathrm{~ms}$, spatial resolution $=1.3 \times 1.3 \times 8.0 \mathrm{~mm}^{3}$, bandwidth $=$ $1502 \mathrm{~Hz} /$ Pixel, partition number $=10$. The acceleration factors were $\mathrm{R}=4$ and 8 , corresponding to scan time $0.76 \mathrm{~min}$ and $0.38 \mathrm{~min}$. The standard $2 \mathrm{D}$ multi-slice ECG-triggering and conventional self-gating methods with the same spatial and temporal resolutions were also conducted for comparison.

\section{Results}

All MR scans were successfully conducted. SparseSG allowed a whole-heart coverage of 3D cine imaging within $1 \mathrm{~min}$, which was much shorter than those of the ECG-triggering and SG methods (Figure 2.b). As the acceleration factor increased, the reconstructed cine images of SparseSG become a little blurry. However, the left ventricle ejection fraction (LVEF) and cardiac structure obtained from SparseSG were in good agreement with those from conventional ECG-triggering and SG methods, even if a high acceleration factor $\mathrm{R}=8$ was used (Figure 2.a\&c).

\section{Conclusions}

An accelerated SG technique, SparseSG, was developed to realize 3D cardiac cine imaging at 3T without ECG and breath-holding. Preliminary in vivo study demonstrated that whole heart coverage of 3D cine imaging can be achieved within $1 \mathrm{~min}$ and the technique has excellent performance compared to the standard ECGtriggering and conventional SG methods. This warrants further evaluation of SparseSG on more volunteers and patients.

${ }^{1}$ University of Science and Technology of China, Hefei, China

Full list of author information is available at the end of the article 
$\mathbf{a}$

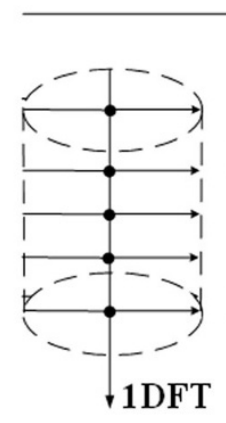

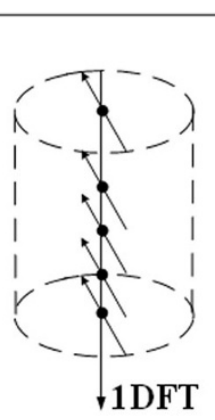
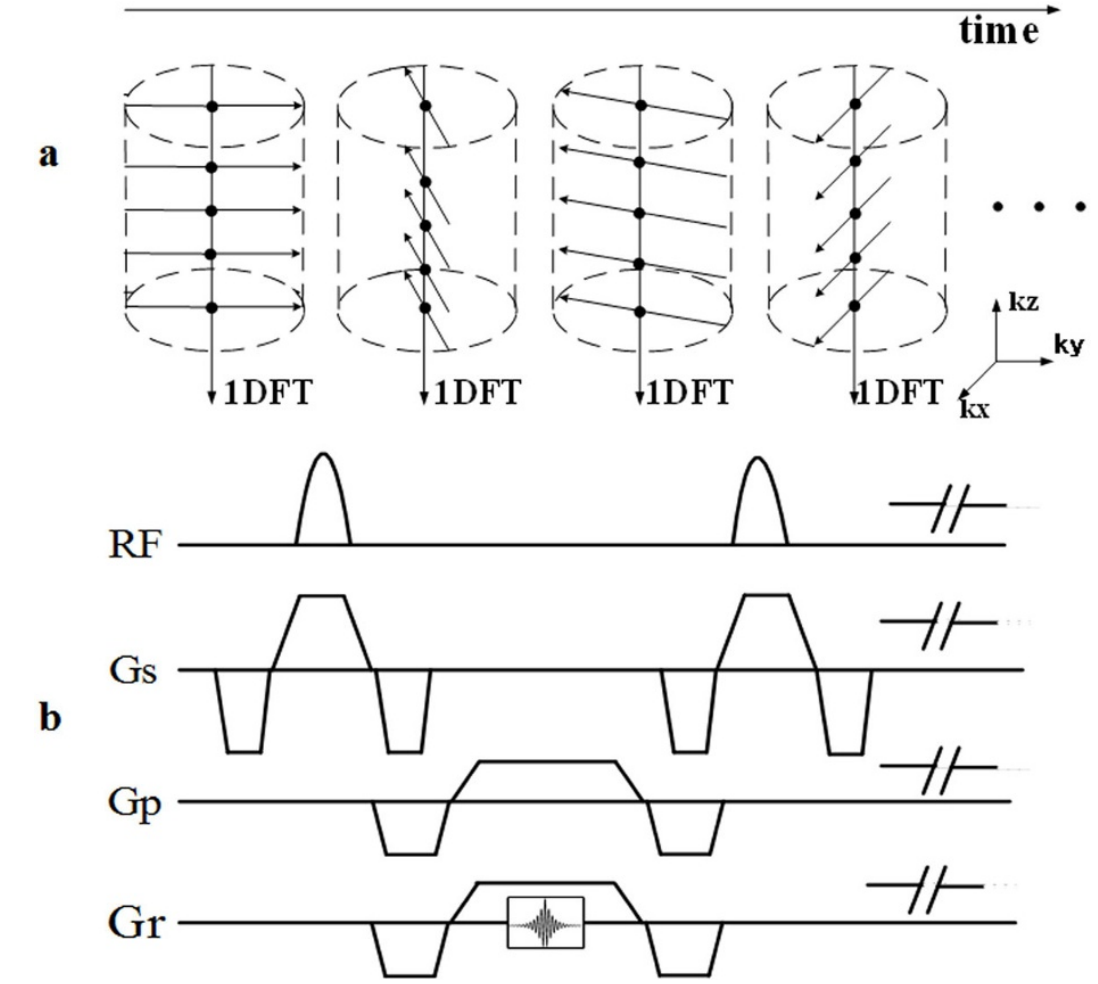

Figure 1 Diagram of the proposed SparseSG technique. (a) A tiny golden angle of $32.039^{\circ}$, instead of $111.246^{\circ}$, was used in the stack-of-stars sampling trajectories, the dot (•) denotes the SG data.(b) the diagram of the TrueFISP sequence with stack-of-stars sampling trajectories.

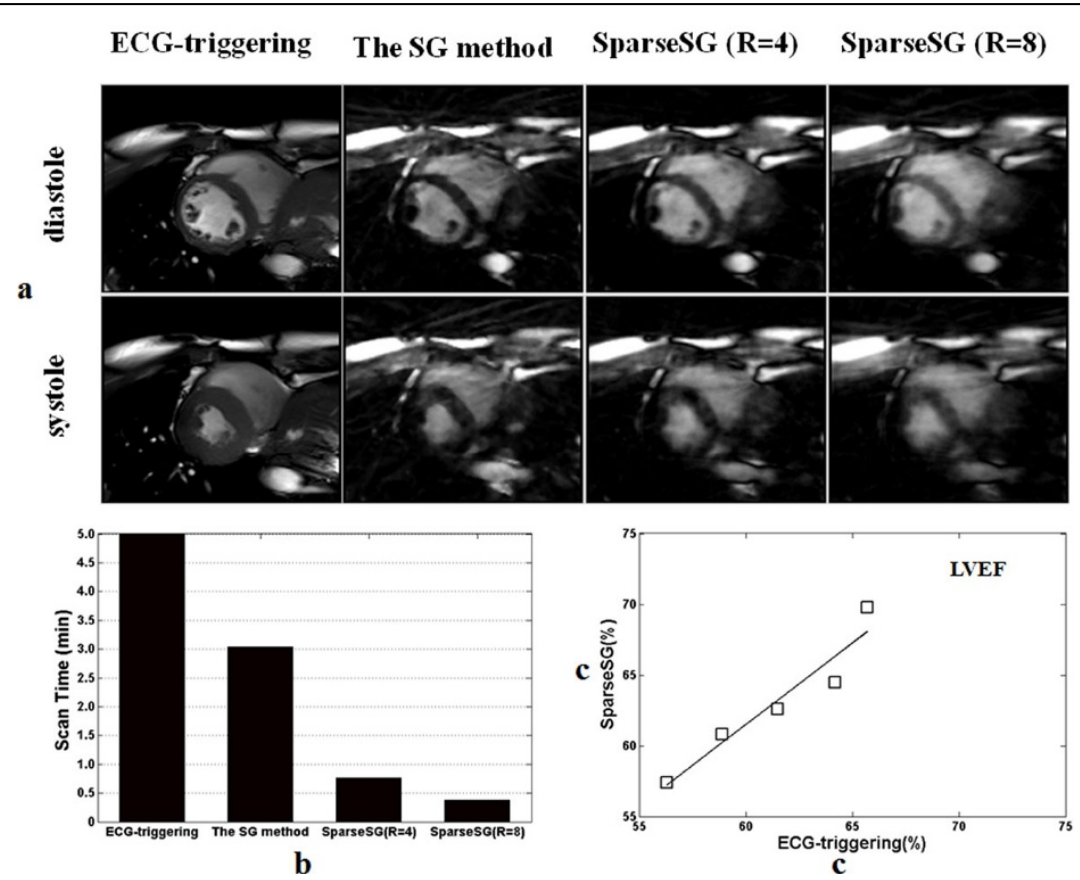

Figure 2 (a) Representative images of the diastole (top row) and systole phases (bottom row) on SA view from a subject. Compared to the standard ECG-triggering and conventional SG methods, cardiac structure obtained by SparseSG were well agreement with those by standard ECG-triggering and conventional SG methods. (b) Whole heart coverage of 3D cine imaging can be achieved within 1 min by SparseSG, which is much shorter than the standard ECG-triggering and conventional SG methods.(c) The left ventricular ejection fraction calculated from the images obtained by SparseSG was well agreement to the standard ECG-triggering method. 


\section{Authors' details}

${ }^{1}$ University of Science and Technology of China, Hefei, China. ${ }^{2}$ Shenzhen Institutes of Advanced Technology, Shenzhen, China. ${ }^{3}$ Guangzhou Panyu Central Hospital, Guangzhou, China. ${ }^{4}$ Cedars-Sinai Medical Center, Los Angeles, CA, USA.

\section{Published: 27 January 2016}

\section{References}

1. Liu J, et al: MRM 2010, 63(5):1230-1237.

2. Wundrak S, et al: 2015 , doi: $10.1002 / \mathrm{mrm} .25831$.

doi:10.1186/1532-429X-18-S1-P305

Cite this article as: Zhang et al:: Accelerated 3D self-gated cardiac cine imaging at $3 \mathrm{~T}$ using a tiny golden angle and compressed sensing.

Journal of Cardiovascular Magnetic Resonance 2016 18(Suppl 1):P305.

Submit your next manuscript to BioMed Central and take full advantage of:

- Convenient online submission

- Thorough peer review

- No space constraints or color figure charges

- Immediate publication on acceptance

- Inclusion in PubMed, CAS, Scopus and Google Scholar

- Research which is freely available for redistribution

Submit your manuscript at www.biomedcentral.com/submit
C Biomed Central 